\title{
Linx
}

Revue des linguistes de l'université Paris X Nanterre

$55 \mid 2006$

Thème et thématisation

\section{L'analyse thème / rhème dans une perspective diachronique}

\section{Bernard Combettes}

\section{(2) OpenEdition}

\section{Journals}

Édition électronique

URL : http://journals.openedition.org/linx/392

DOI : $10.4000 /$ linx.392

ISSN : 2118-9692

\section{Éditeur}

Presses universitaires de Paris Nanterre

\section{Édition imprimée}

Date de publication : 1 décembre 2006

Pagination : 75-89

ISSN : 0246-8743

\section{Référence électronique}

Bernard Combettes, «L'analyse thème / rhème dans une perspective diachronique », Linx [En ligne], 55 | 2006, mis en ligne le 22 février 2011, consulté le 30 avril 2019. URL : http:// journals.openedition.org/linx/392 ; DOI : 10.4000/linx.392 


\title{
L'analyse thème / rhème dans une perspective diachronique
}

\author{
Bernard Combettes \\ Nancy 2 et UMR-ATILF
}

L'analyse de la Perspective fonctionnelle de la phrase, telle qu'elle a été développée par l'École de Prague, met en œuvre, entre autres caractéristiques, deux principes dont l'application dans le domaine diachronique est d'une pertinence certaine. La mise en relation du niveau informatif, où s'établit la hiérarchie des divers constituants sur l'échelle de thématicité ou de rhématicité, avec l'organisation syntaxique de l'énoncé pour assurer le codage des degrés du dynamisme communicatif est directement concernée par les changements qui peuvent affecter l'ordre des unités ; dans la mesure où l'évolution affecte l'existence de tel ou tel schéma de phrase ainsi que les contraintes plus ou moins fortes qui régissent les faits de linéarisation, la structure informationnelle de l'énoncé est évidemment visée par ces éventuelles modifications. Par ailleurs, la prise en compte de la dimension textuelle dans l'analyse des différentes progressions thématiques, au niveau des grandes unités discursives, mérite également d'être replacée dans une perspective diachronique : il parait pertinent d'examiner si les transformations du niveau syntaxique s'accompagnent de la mise en œuvre de nouvelles structurations des séquences textuelles. Ce sont ces deux points que nous développerons ici en nous appuyant sur l'examen de textes de l'ancien et du moyen français. C'est en effet au cours de cette transition qui conduit au système linguistique représenté dans les corpus des XIVe et XVe siècles que s'opèrent les grands changements concernant l'ordre des constituants de l'énoncé ; il semble légitime de se demander comment évolue, durant cette période, l'interface entre les faits de position et la dynamique informationnelle et d'observer également si ces modifications vont de pair avec des changements dans le domaine de la cohérence discursive. 


\section{Schémas syntaxiques et hiérarchisation thème / rhème}

\subsection{Schéma à verbe second}

Une des principales caractéristiques qui permettent de considérer que le système syntaxique de l'ancien français laisse la place à celui du moyen français réside dans la disparition progressive des propriétés inhérentes au schéma de base à verbe second. Ce changement, qui ne concerne pas que des faits de linéarisation, mais, plus profondément, pourrait-on dire, la hiérarchisation des divers groupes syntaxiques, l'ordre : déterminant / déterminé, est déjà bien entamé, du moins pour certains faits de langue, durant la période de l'ancien français (cf. Buridant, 1987 ; Bauer, 1995) ; en ce qui concerne l'ordre des constituants, toutefois, la séquence qui réserve la deuxième zone de l'énoncé à la forme verbale demeure très majoritaire, qu'il s'agisse de la progression à ordre direct SVX ou du schéma à postposition du sujet $\mathrm{XV}(\mathrm{S})$, cette systématisation étant peut-être due aux aspects formulaires et archaïsants de la prose narrative. Se pose ainsi la question de l'organisation du niveau informatif, des relations que ce type de structuration syntaxique entretient avec la hiérarchisation des unités sur l'échelle du dynamisme communicatif. Quelles sont les contraintes qu'un système à verbe second entraîne dans ce qu'on peut considérer comme le marquage des degrés de thématicité ou de rhématicité d'un constituant ? Quelles sont par ailleurs, d'un point de vue diachronique, les conséquences, pour l'organisation de l'information, de l'évolution vers un système dans lequel le verbe ne se voit plus attribuer une position fixe dans la proposition? Comme nous allons le voir, ce ne sont pas seulement les réalisations de la linéarisation de surface mais, plus profondément, les principes du codage du niveau informatif qui se trouvent atteints par les modifications des règles de position. Observons quelques caractéristiques de la situation la plus courante en ancien français. Les énoncés suivants, (1) à (5), qui sont tous construits sur le schéma syntaxique à verbe second, ont pour point commun de présenter en zone initiale un constituant, groupe prépositionnel à fonction circonstancielle pour les trois premiers, complément essentiel pour le dernier d'entre eux, qui se rattache de façon très nette au contexte antérieur par une relation anaphorique. Si le début de phrase, l'unité syntaxique qui précède le verbe, relève ainsi clairement de la partie thématique, il est facile de constater que l'ensemble VS(X) est loin de présenter la même homogénéité. En (1), par exemple, ni le verbe ni le sujet postposé, malgré l'emploi du déterminant défini (la noise), qui peut être considéré ici comme un article de "notoriété », ne peuvent être inférés à partir du contexte ; c'est l'ensemble qu'ils constituent qui remplit la fonction de rhème propre sur l'échelle des degrés du dynamisme communicatif :

1- $\quad$ A ceste parole leva la noise el palés (La Mort Artu, XIII $\left.{ }^{\mathrm{e}} \mathrm{s}.\right)$

[A cette parole, la dispute s'éleva dans le palais]

En revanche, dans :

2- $\quad$ En tel maniere dura li estris jusqu'à vespres

[Le combat dura de cette façon jusqu'au soir] 
c'est l'unité formée par la forme verbale (dura) et le circonstant (jusqu'à vespres) qui est dotée de ce rôle de rhème propre, le sujet ne faisant que renvoyer à un référent saillant. Il en va de même dans :

3- $\quad$ Dont esgarda li empereres un bourgois qui (...)

(Helcanus, XIII s.) [Alors l'empereur regarda un habitant du bourg qui...]

où la présence du sujet immédiatement après le verbe conduit à la discontinuité du rhème esgarda ... un bourgois. Dans la plupart des cas, l'interprétation de l'organisation de l'information, en l'absence de marquage syntaxique biunivoque, est fonction de la plus ou moins grande accessibilité du référent auquel renvoie le syntagme sujet. Ainsi, en (4) la saillance du référent, qui pourrait d'ailleurs se traduire par la non expression du sujet, conduit-elle à interpréter le verbe s'accordent comme le focus de l'énoncé :

4- A cele chose s'accordent ambedui

[Tous deux s'accordent à cette chose]

alors que, dans :

5- $\quad$ A cest conseil survint Hector

(La Mort Artu, XIII" s.)

[A ce conseil survint Hector]

le référent, facilement identifiable, doit cependant être réactivé, réintroduit dans le fil du discours, dans la mesure où il n'a pas été mentionné dans le contexte proche. Ces quelques exemples témoignent de la dissymétrie que comporte le système de marquage de la structure informationnelle de l'ancien français : l'accent est mis sur la délimitation du thème, non seulement comme l'élément occupant la position initiale, mais aussi comme le constituant qui remplit toute la zone préverbale. En ce sens, le verbe joue un rôle de démarcateur dans la progression thématique de l'énoncé, la zone rhématique, syntaxiquement soumise à la contrainte du schéma XVSX, ne reflétant pas de façon biunivoque les degrés de dynamisme communicatif.

Ce que l'on peut considérer comme une certaine rigidité dans l'organisation linéaire de l'énoncé et, par contrecoup, dans la hiérarchisation de la structure informative, ne permet pas non plus de marquer de façon claire les cas de contraste ou d'emphase, qui auront plus tard un traitement spécifique par la création de constructions présentatives de type c'est ... que. Dans l'extrait suivant, c'est le parallélisme entre les deux phrases (si li mist nom la reine / li mist nom Keu le senechal...) et, surtout, l'enchaînement avec le contexte antérieur qui permettent d'interpréter les sujets inversés comme des rhèmes propres ; le fait que le syntagme li mist nom reprend ot il nom et ne peut être considéré comme une information nouvelle conduit à analyser le groupe sujet comme focus, mais on remarquera que le circonstant de lieu (devant Estreberes) pourrait également remplir cette fonction (c'est devant Estreberes que la reine lui donna ce nom), dans la mesure où l'ordre des constituants impose de placer le sujet dans la proximité immédiate du verbe ou de la locution verbale :

6- Et pour la grant prouesse qu'il avoit quant il estoit eschauffé, ot il nom Sagremors li Desreez. Si li mist nom la reine devant Estreberes, (...) Et pour la maladie qui si souvent lui advenoit, li mist nom Keu li senechal Sagremor le Mort Jeun. (id.) 
[Et pour le grand courage qu'il avait quand il était irrité, il eut pour nom Sagremort le Desréé. Et ce fut la reine qui lui donna ce nom devant Estreberes (...) Et pour la maladie qui le prenait si souvent, ce fut Keu le sénéchal qui lui donna le nom de Mort Jeun]

Il semble difficile, pour appréhender correctement le fonctionnement de ces contraintes qui s'exercent au niveau informatif, de ne pas prendre en compte l'opération de prédication. Tout se passe en effet comme si la linéarisation des unités se trouvait commandée par des facteurs d'ordre différent : la tendance à organiser la progression thématique en fonction de l'échelle thème / rhème se voit contrecarrée par une autre contrainte, qui consiste à réaliser dans une même unité de traitement la prédication et l'identification du référent auquel renvoie le groupe sujet; le système à verbe second avec postposition du sujet permet de généraliser ce principe, de lui donner en quelque sorte la priorité sur l'application de règles qui prendraient en compte toute la gamme des degrés de la perspective fonctionnelle.

\subsection{Schémas (X) Sujet (X) Verbe}

Cette structuration de la composante informationnelle, qui repose sur une dichotomie thème / rhème mettant en valeur le thème propre et neutralisant les degrés intermédiaires de l'échelle du dynamisme communicatif, va se trouver modifiée par les modifications profondes qui affectent la syntaxe de position durant la période du moyen français. Nous ne traiterons pas ici la question complexe des enchainements des causes et des effets. On peut en effet s'interroger sur la nature et la hiérarchisation des facteurs qui ont conduit à la perte du schéma à verbe second comme construction de base de la prose narrative. Que l'on mette en avant des raisons d'ordre syntaxique, liées à l'équilibre interne du système, ou des raisons d'ordre discursif, dépendant de l'évolution des structures textuelles, il semble difficile de ne pas prendre en compte, dans une perspective fonctionnaliste, la composante informationnelle, la conception même de la cohérence discursive, dont l'évolution va de pair avec celle des marques linguistiques. Dans ces changements qui affectent l'interaction du niveau syntaxique et du codage des degrés de dynamisme communicatif, deux faits marquants sont à noter : d'une part, le marquage privilégié, dans le système à verbe second, du syntagme qui est à interpréter comme le thème propre se voit remplacé par celui du rhème propre, d'ordinaire constitué du verbe et de certains compléments ; la zone finale de l'énoncé devient ainsi le lieu discriminant, là où les énoncés de l'ancien français ne hiérarchisaient guère l'organisation informationnelle. Le «recul» du verbe entraîne d'autre part la création de nouvelles zones en position préverbale, en particulier entre le syntagme sujet et l'élément verbal ; prédications secondes et rhèmes secondaires, sous la forme de compléments de manière, peuvent ainsi être nettement séparés du thème et du rhème propres. La réorganisation de la ligne de l'énoncé ne correspond donc pas seulement à un changement de surface dans la place des diverses unités, mais, plus profondément, à une modification dans le type de codage dévolu, en ce qui concerne le dynamisme communicatif, aux constructions syntaxiques. C'est ainsi que l'on assiste à la grammaticalisation d'un schéma SXV, qui autorise l'insertion, entre le sujet et le verbe, de constituants dotés d'un faible degré informatif et laissant attendre le rhème 
propre. Dans les exemples suivants, des appositions, des constructions absolues, des adverbes de manière occupent cette position intermédiaire :

7- $\quad$ Alors Jehan de Saintré, comme humble, doux et gracieux, incontinent devant le roi à genoux se jeta (A. de La Sale, Jehan de Saintré, 1456)

[alors Jean de Saintré, en homme bumble, doux et gracieux, immédiatement se jeta à genoux devant le roi]

8- $\quad$ Alors Louis, l'escu au col, la lance au poing, saillit sur son destrier (Histoire des Seigneurs de Gavre, 1456)

[alors Louis, l'écu pendu au cou, la lance au poing, sauta sur son cheval]

Dans la mesure où la zone initiale de l'énoncé est toujours la place privilégiée pour les adverbiaux cadratifs - on relèvera l'emploi de l'adverbe alors dans les deux exemples précédents -, et où l'inversion du sujet n'est plus systématique, il n'est pas étonnant que la progression XSXV (X) apparaisse fréquemment dans les textes. Les différents degrés de dynamisme communicatif sont ainsi attribués à des places spécifiques, le groupe sujet jouant le rôle de pivot, de point de repère, dans ce type d'organisation, rôle que tenait le verbe dans le système de l'ancien français. Les constituants qui précèdent le sujet nominal sont ainsi interprétés comme relevant de la partie thématique, alors que ceux qui apparaissent dans le contexte de droite appartiennent au rhème, qu'il s'agisse du rhème propre ou de rhèmes secondaires. Le passage suivant montre bien cette fonction de délimitation que remplit le syntagme sujet :

9- $\quad$ Mais au departir l'un de l'autre, en toutes façons Saintré per à per et à sa dextre le convoya. (A. de La Sale, Jehan de Saintré, 1456)

[mais lorsqu'ils se séparèrent, Saintré l'accompagna d'égal à égal à sa droite de toutes les manières possibles]

La fixation du syntagme objet à la suite du verbe étant loin d'être totalement réalisée en moyen français, le début de phrase peut également contenir un constituant essentiel, la progression thématique s'effectuant toujours de la même manière, avec une délimitation de la partie thématique et de la partie rhématique marquée par le groupe sujet, comme dans :

10- Alors tous deux, qui à genoux estoient, le roi commanda à lever

[alors le roi leur ordonna à tous deux, qui étaient à genoux, de se relever]

Dans une comparaison de la structure informationnelle que présentent les textes d'ancien français et de celle qu'adopte le moyen français, il conviendrait par ailleurs de ne pas limiter l'observation, comme nous le faisons ici, aux faits de position; la question de la densité de l'information devrait être également prise en compte. Il semble en effet que les changements syntaxiques, s'ils autorisent une présentation plus affinée et plus précise des degrés de dynamisme communicatif, s'accompagnent aussi d'un accroissement du nombre des « unités d'information » que contient l'énoncé (cf. Chafe, 1994 ; Lambrecht, 1984, 1987), en particulier des prédications secondes, qui se substituent, dans bon nombre de cas, à des prédicats qui, 
en ancien français, étaient portés par des subordonnées (cf. Combettes, 2000). Il serait également nécessaire d'examiner la question, difficile à traiter pour les états de langue anciens, de ce que l'on peut appeler les «paliers de traitement» pertinents pour les phénomènes relevant du niveau informatif ; la comparaison des deux passages suivants permet de constater facilement la grande différence qui sépare un texte fondé sur la parataxe, constitué d'une succession de propositions correspondant à une prédication unique, et un texte dans lequel les prédications secondes (le tenant, sa poitrine sur la sienne) viennent briser cette régularité de l'itération des structures à verbe second et conduisent par exemple à une nouvelle mention du sujet thème (damps Abbés) :

11- Einsi se partirent de l'ost Guillaume de Champlite et Villehardouin. Et emmenèrent bien cent chevaliers avec eux. Et entrerent en la terre de la Morée. Michalis oï qu'ils estoient à si peu de gens en la terre. Si amassa gens. Et ce fut une merveille de gens. Et chevaucha après eux (...)

(G. de Villehardouin, La conquête de Constantinople, c. 1210)

12- Lors damps Abbés estent sa jambe, et par dedens la lie à celle de Saintré, puis le trousse tellement que à terre l'abattit, et en le tenant sous lui, sa poitrine sur la sienne tout envers, alors s'escria damps Abbés et à Madame dit... (A. de La Sale, Jehan de Saintré, 1456)

[Alors messire Abbé tend la jambe et la lie par derrière à celle de Saintré, puis le renverse si brusquement qu'il le jeta à terre, et en le tenant sous lui, sa poitrine contre la sienne, alors messire Abbé s'écria et dit à Madame...]

\section{Progressions thématiques}

Ces modifications, qui concernent l'organisation du dynamisme communicatif et qui, comme nous avons essayé de le montrer, affectent le système même de codage, doivent être prises en considération à un niveau plus large, celui de la construction de la cohérence des séquences discursives. Le changement de statut des schémas syntaxiques à verbe second a des répercussions sur la structuration informationnelle du texte, qu'il s'agisse du traitement des enchaînements thématiques ou, plus généralement, de la gestion de la connaissance partagée, de l'introduction des référents nouveaux, de la réactivation de référents déjà évoqués, par exemple. La répartition de l'information que favorise le système syntaxique de l'ancien français s'accompagne en effet, au niveau textuel, de la généralisation de deux progressions thématiques caractéristiques de la prose narrative de cette époque. Dans la mesure où la hiérarchisation des unités informatives donne la priorité à l'identification du thème, il n'est pas étonnant que la progression à thème constant et la progression à thème linéaire soient de loin les mieux représentées. Dans le premier cas, le maintien d'un thème identique permet d'ordinaire de rapporter la succession des actions d'un personnage ; dans ces séquences narratives, la référence au thème fait alterner l'emploi des pronoms anaphoriques et la non expression du sujet : 
13- Et neporec tant a allé que il vint a une croiz de pierre qui ert au departement de deus voies en une gaste lande. Et il regarde la croiz quant il fu pres et voit par dejoste un perron de marbre ou il avoit lettres escrites (...) et il resgarde vers la croiz et voit une chapelle mout ancienne (...) et troeve a l'entree unes prones de fer (...) et voit la dedenz un autel qui (...)(La Queste del Saint Graal, XIIIe s.) [et cependant il a tant chevauché qu'il arrive à une croix de pierre qui était à la séparation de deux chemins sur une lande déserte. Et il regarde la croix quand il en est près et voit à côté un bloc de marbre où il y avait des lettres écrites (...) et il regarde vers la croix et voit une chapelle très ancienne (...) et il trouve à l'entrée des barreaux de fer (...) et il voit à l'intérieur un autel qui ....]

Comme nous le verrons plus loin, la progression à thème linéaire est privilégiée dans la construction du texte descriptif. L'expression du thème peut s'opérer de façon relativement variée: pronom sujet, ou anaphore nominale en fonction de sujet, d'objet, ou de circonstant. On notera également le rôle des pronoms relatifs qui assurent la liaison avec un antécédent ; le développement, en moyen français, des formes de type lequel, qu'il s'agisse des pronoms ou des déterminants, ne fait que renforcer cette tendance. Dans le passage suivant, on relèvera l'emploi du syntagme sur lequel drap d'or et du pronom onquel:

14- Ou ranc des cinq dessus nommez estoit une tombe, ou pour plus vray parler ung cercueil, car il n'estoit fait que de boys seulement, neantmoins qu'il avoit par dessus ung drap d'or (...) sur lequel drap d'or avoit une grant blanche croix (...) et ou chief de la tombe avoit ung carreau de satin (...) ouquel avoit lettres brodees. (René d'Anjou, Le Livre du Cuer d'Amours espris, 1457)

[à la suite des cinq nommés ci-dessus était une tombe ou, à dire vrai, un cercueil, car il n'était fait que de bois, bien qu'il y ait au dessus un drap d'or (...) sur lequel drap d'or il y avait une grande croix blanche (...) et à la tête de la tombe il y avait un coussin de satin (...) sur lequel il y avait des lettres brodées]

\subsection{Introduction des référents nouveaux}

Les deux progressions thématiques que nous venons d'illustrer présentent un point commun dans leur fonctionnement au regard de la cohérence discursive et, plus particulièrement, dans les opérations qui concernent l'introduction d'un référent nouveau dans le texte. Elles permettent en effet, dans la mesure où la zone thématique est clairement assimilée à celle qu'occupe le premier syntagme de l'énoncé, de placer en position postverbale les constituants qui renvoient à des référents qui apparaissent pour la première fois dans le flux discursif ou qui nécessitent une réactivation ; se trouve ainsi respectée la tendance évoquée plus haut, qui semble systématisée dans la prose narrative de l'ancien français, à traiter successivement, dans la linéarisation des éléments constitutifs de l'énoncé, l'opération de prédication et celle d'identification. Tout se passe comme si cette dernière ne pouvait intervenir avant que ne soit établi le prédicat principal dont le syntagme renvoyant au référent nouveau saturera 
éventuellement la valence. Cette contrainte se laisse clairement percevoir dans le cas de la séquence d' «introduction» du sujet : X + Verbe + Sujet, mais peut être également étendue aux autres constituants, qu'il s'agisse de compléments essentiels ou de circonstants. Cette tendance générale se réalise dans deux grands types de schémas syntaxiques à ordre V2 : Sujet + V (X) et X + V (Sujet). Dans le premier cas, le sujet renvoie ordinairement à un référent animé qui entre dans une progression à thème constant ; c'est en quelque sorte par l'intermédiaire de cet actant que s'opère l'introduction des référents nouveaux. Les prédicats comportent alors, de façon assez naturelle, des verbes de perception ou des verbes de mouvement, qui sont accompagnés de compléments renvoyant à la réalité à décrire. Ainsi, dans les deux extraits suivants :

15- quant Melyanz se fu partis de Galaad, il chevaucha jusques à une forest ancienne qui duroit bien deus jornees, et tant que il vint l'endemain à heure de prime en une praerie. Si voit ou milieu dou chemin une chaiere belle et riche ou il avoit une coronne d'or trop belle; et devant la chaiere avoit plusors tables remplies de biaus mangiers

\section{La queste del Saint Graal}

[quand Meliant se fut séparé de Galaad, il chevaucha jusqu'à une forêt (...) si bien qu'il arriva à une prairie. Et il voit au milieu du chemin un trône où il y avait une couronne d'or (...) et devant le trône il y avait plusieurs tables ...]

16- Quant il a chevauchié jusque à midi, si voit devant lui fors dou chemin une petite maison. Il torne celle part, car il set bien que ce est hermitages. Quant il est jusque la venuz, si voit une petite chapelle en une petite maison. Et devant, à l'entrée, se seoit uns vieil hom vestu de robe blanche en semblant d'hom de religion... (id.)

[quand il a chevauché jusqu'à midi, il voit devant lui une petite maison. Il s'y dirige (...) Quand il y est arrivé, il voit une petite chapelle (...) Et devant, à l'entrée, était assis un vieil bomme ...]

les formes verbales chevaucha, vint se combinent-elles avec voit pour permettre l'introduction de syntagmes indéfinis (une forest ancienne, une praerie, ...) en zone postverbale. On notera que ces constituants compléments, s'ils ne renvoient pas à des référents qui joueraient un rôle important dans la narration, - il s'agit essentiellement de non animés traduisant des circonstances spatiales - peuvent toutefois être repris pour assurer l'enchaînement discursif, doublant, d'une certaine manière, la progression à sujet thème constant (une petite maison ... celle part ...). Le deuxième schéma, $\mathrm{X}+$ Verbe + Sujet / Y, avec un constituant autre que le sujet en zone initiale, se conforme également à la progression Thème + Rhème et contient d'ordinaire un verbe statif, l'impersonnel (y) avoir ou les formes passives étant de loin les mieux représentés; les enchaînements s'organisent alors suivant une progression de type «linéaire », le nouvel 
élément ainsi introduit constituant à son tour le thème dans le contexte postérieur, comme dans l'extrait suivant, où les groupes une grosse colonne et une pierre sont repris sous la forme de circonstants (sur le bout de cette colonne, sur cette pierre) :

17- aprés devant chu moustier de Sainte Sophie, avoit une grosse colombe qui bien avoit trois brachies à un homme de grosseur, et si avoit bien cinquante toises de haut; si estoit faite de marbre et puis de coivre par dessus le marbre, et estoit molt bien liée de bonnes andes de fer. Lassus, sur le bout de chelle colombe, si avoit une pierre qui bien avoit quinze piés de lonc et autant de lé. Sur chelle pierre si avoit un empereur jeté de coivre, qui tendoit sa main vers paienisme; et avoit letres sur lui escrites qui disoient que (...)

(Robert de Clari, La conquête de Constantinople, c. 1210)

[devant ce monastère de Sainte Sophie, il y avait une grosse colonne qui (...) En baut, au sommet de cette colonne, il y avait une pierre qui (...) Sur cette pierre il y avait un empereur en cuivre qui ...]

Les deux types d'organisation peuvent évidemment se trouver combinés dans le même passage. La description qui suit fait alterner des verbes de perception (aperçut, vit) et des verbes qui localisent les référents (pendait, (y) avait) :

18- quant ilz eurent passé la première porte et furent sous le portail, le Cueur apperceut en la voulte du portail pendue une corbeille faite d'osier, ancienne et de vieille façon, laquelle pendoit à une chaigne d'or grosse comme le bras. Et en regardant qu'il faisoit ladicte corbeille, s'arresta et vit unes forces de fer enroullees qui estoit de la grandeur d'un pié et demi (...) Entre le taillant desquelles forces avoit une grosse poignée de cheveulx noirs comme terre et long d'une toise ou plus (...). Si n'eut gueres regardé la qu'il apperceut encores joignant de la dicte force, laquelle pendoit à un gros croc d'or, si vit une bride de cuir, une selle et ungs esperons dorez (...) Et plus avant encores y avoit une ymaige de boys grande et enfumée (...)

\section{René d'Anjou, Le Livre du Cuer d'Amours Espris}

[le Cour aperçut sous la voûte du portail une corbeille pendue (...) laquelle pendait à une chaine (...) Et pendant qu'il regardait cette corbeille, il s'arrêta et vit des ciseaux (...) Entre les lames de ces ciseaux, il y avait une grosse poignée de cheveux (...) il aperçut encore, à côté de ces ciseaux, une bride de cuir, une selle et des éperons dorés (...) Et plus en avant il $y$ avait une image de bois ...]

On pourra remarquer, dans ce type de progression, le rôle joué par la subordination relative, qui fait coïncider le thème avec le relatif qui ouvre la proposition, le relatif du type lequel jouant d'ailleurs davantage un rôle d'anaphorique que de véritable subordonnant.

En ce qui concerne les relations qui peuvent être établies entre le niveau thématique et la typologie textuelle, il convient de noter le statut particulier de la 
description ou, plus généralement, des séquences de second plan. Dans le cas de l'ordre Sujet + Verbe $(\mathrm{X})$, tout se passe en effet comme si les parties descriptives se trouvaient en quelque sorte dépendantes du premier plan, subordonnées à la narration; elles ne constituent pas des ensembles, des unités discursives, qui auraient leur structuration propre, mais des ajouts, des précisions "circonstancielles", qui ne peuvent apparaître que comme morcelées, insérées dans le fil du récit, dans la mesure où elles sont étroitement liées aux prédicats de perception ou d'action qui constituent la trame du premier plan. Il en va autrement avec le deuxième type de progression. Les passages descriptifs peuvent former des unités textuelles assez étendues (cf. 17), la progression linéaire permettant de passer d'un élément du second plan à un autre, sans intervention de prédicats qui renverraient à un déroulement chronologique. De tels passages peuvent néanmoins difficilement rendre compte d'impressions d'ensemble, aller par exemple d'une vision générale à la relation de détails, dans la mesure où ils consistent fondamentalement dans les constructions de chaines référentielles renvoyant à des éléments qui, dans la réalité décrite, entretiennent des rapports de proximité. De la même façon que les passages de premier plan reflètent, par un effet d'iconicité, la dimension chronologique d'une séquence d'événements, les parties de second plan « respectent » la disposition spatiale des objets représentés. La description, lorsqu'elle n'est pas subordonnée au récit, adopte ainsi une organisation identique à celle de la narration. Nous allons voir que les types de plans sont l'objet, durant la période du moyen français, d'une évolution qui va dans le même sens.

\subsection{Progressions à thèmes dérivés}

Les progressions thématiques que nous venons d'illustrer se caractérisent par ce que l'on pourrait appeler une cohérence «étroite »; chaque énoncé présente, en zone initiale, un référent - actant ou circonstant - qui est doté d'un degré élevé d'accessibilité, dans la mesure où il est ordinairement évoqué dans le contexte immédiat. L'utilisation fréquente, dans les passages de premier plan, dans les séquences narratives, d'outils de liaison comme si ou et ne s'oppose pas à cette tendance. La non expression du sujet qui va habituellement de pair avec ce type d'enchainement correspond en effet à une continuité thématique forte, le thème constant se réalisant dans le sujet zéro. Les textes de moyen français, du moins à partir du $X V^{e}$ siècle offre toutefois l'exemple d'une cohérence plus large, qui ne repose plus sur des enchaînements interphrastiques immédiats, mais sur des relations qui mettent en jeu l'ensemble de la séquence discursive. C'est alors la progression à « thèmes dérivés » ou à hyperthème qui se substitue à l'organisation à thème constant ou à thème linéaire. Le principe de la progression à thèmes dérivés n'est certes pas ignorée de l'ancien français, mais dans les cas où elle survient, elle se présente comme une variante de la progression linéaire, deux référents, ou des sous-parties d'un référent, se trouvant utilisés successivement comme thèmes dans le contexte de droite. Le moyen français conserve évidemment cette possibilité, comme dans ce passage de Commynes :

19- [L'armée dudict duc estoit en deux parties.] L'une menoit le mareschal de Bourgogne (...) L'autre partie estoit avecques ledict duc.

(Commynes, Mémoires, 1495) 
[L'armée de ce duc était formée de deux parties. Le maréchal de Bourgogne conduisait l'une (...) L'autre partie était avec le duc]

Ce qui est nouveau, c'est l'emploi d'énoncés dont aucun référent n'est accessible dans le contexte proche, mais qui se présentent comme entièrement rhématiques et sont interprétés comme des prédicats dérivés d'un prédicat plus général qui les annonce, les introduits. Il faut souligner que, d'une part, les liaisons ne s'opèrent plus par l'intermédiaire de référents particuliers et que, d'autre part, elles mettent en jeu l'ensemble de l'unité textuelle. Les deux passages suivants, tirés des Chroniques de Jean Molinet, illustrent bien ce nouveau type de cohérence :

20- Franchois, tous avisés de leur faict, besongnèrent à leurs pieces ; embusches se descouvrirent; eschielles furent dressiez; gens d'armes gaignèrent les murs. (Jean Molinet, Chroniques, 1490)

[Les Français, avertis de leur (= des ennemis) entreprise, se mirent à l'ouvrage; des embuscades se préparèrent; des échelles furent dressées; des hommes d'armes gagnèrent les murs]

21- Dès l'année précédente, avoit esté le tempz d'yver tant aspre et angoisseux que (...) Petis enfans estoyent trouvéz mors en leurs repos et berceaux, pluseurs gens à cheval s'engeloyent par les champz (...) Pelerins, pietons, bosquillons et ceulx qui hantoyent les bois rendirent leurs esperis (...) Les oyseaulx du ciel estoyent recoeulliéz morz (...) ; pluseurs arbrez morurent geléz et steriles. (id.) [dès l'année précédente le temps d'hiver avait été si rude et si dur que (...) Les petits enfants étaient trouvés morts dans leurs berceaux, plusieurs qui allaient à cheval étaient pris par le gel dans la campagne (...) Les pèlerins, ceux qui allaient à pied, ceux qui vivaient dans les bois rendirent l'âme (...) Les oiseaux du ciel étaient ramassés morts (...); plusieurs arbres moururent gelés et stériles]

En (20), le prédicat initial (besognèrent à leurs pièces) renvoient à un ensemble d'activités dont le détail est en quelque sorte donné dans les trois propositions qui constituent la suite de la description. En (21), ce sont les conséquences de l'hiver rigoureux qui sont énumérées après l'hyperthème du début du texte (le temps d'yver tant aspre que ...). En ce qui concerne l'interface syntaxe / niveau informationnel, on peut noter que l'ordre direct, SVX, avec sujet exprimé, voit son statut se modifier: il n'implique plus une décomposition de l'énoncé en deux parties nettement distinctes, en une zone thématique et une zone rhématique, mais peut correspondre à une unité qui relève entièrement du rhème, ou, dans une autre terminologie, à une proposition «all focus », modification importante qui doit être mise en relation, même s'il est difficile de déterminer l'ordre exact des causes et des effets, avec les changements qui affectent la structuration discursive. Cette évolution est évidemment très progressive, la possibilité du schéma à verbe second se maintenant, pendant longtemps encore, dans bon nombre de textes. Ainsi dans l'exemple (19), cité plus haut, qui, il est vrai, est d'un type relativement différent dans la mesure où la progression à hyperthème met en jeu des syntagmes nominaux (l'une, l'autre partie) et non des prédicats, la première phrase est-elle construite sur le schéma XVS, alors que la deuxième correspond à SVX. 
Dans les textes d'ancien français, les passages de second plan apparaissaient comme étroitement dépendants du premier plan. L'évolution que nous venons de décrire, qui exploite pleinement les ressources de la progression à thèmes dérivés, ne se limite pas aux séquences de premier plan ; comme le montrent les extraits que nous venons de citer, les descriptions sont concernées au premier chef par ce changement qui met en jeu une autre conception du rôle de la linéarisation dans la cohérence textuelle. La progression thématique prend ainsi pour point de départ l'ensemble de la situation à décrire et chacun des énoncés successifs rend compte d'un aspect de la réalité sans qu'il y ait nécessairement contiguité entre les éléments sélectionnés. Dans l'extrait suivant, si la première phrase est construite sur un schéma à verbe second qui introduit le référent nouveau par l'intermédiaire d'un verbe de perception, comme dans les exemples de passages descriptifs que nous avons cités plus haut, les autres propositions du texte, à ordre SVX, renvoient à diverses sous-parties de la réalité décrite, qui sont d'ordre relativement différent ; on notera d'ailleurs l'emploi de la locution d'autre part, qui souligne, si l'on peut dire, la solution de continuité :

22- Si virent lors l'air net et pur sans vent et sans nuée, et le jour gagnoit la nuit fortment, en soi esclardissant par façon que la lune n'avoit clarté qui peust plus le cler jour surmonter, et ja les oiselets s'appeloient l'un l'autre. D'autre part la

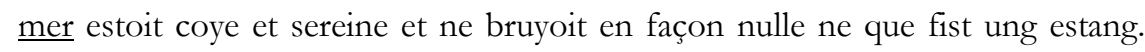
Les mouettes aussi commencerent à voler par dessus la marine, et d'autres si trottoient sur le sablon menu que beau les faisoit veoir. Le jour tant s'efforça qu'il envoya coucher la lune et les estoiles, si que plus nulles ou ciel n'apparaissoit.

(René d'Anjou, Le Livre du Cuer d'Amours Espris, 1457)

[ils virent alors l'air net et pur (...) et le jour gagnait sur la nuit (...) de sorte que la lune n'avait de clarté assez forte pour (...) et déjà les oiseaux s'appelaient les uns les autres. Par ailleurs la mer était calme (...) Les mouettes aussi commencèrent à voler (...) et d'autres trottaient sur le sable (...) Le jour fit tant d'efforts que ....

Ce type d'organisation accentue l'autonomie des parties de second plan, même si certains indices, comme l'hétérogénéité temporelle, avec l'alternance de l'imparfait et du passé simple, montrent que la description n'est pas encore totalement détachée des structures caractéristiques du premier plan narratif.

Dans ce mouvement qui concerne à la fois les structures syntaxiques et les types de progressions thématiques, il faut relever une configuration intéressante, qui pourrait être considérée comme une situation intermédiaire entre le système de l'ancien français, à cohérence étroite, et ce nouveau système dans lequel les relations s'établissent à un niveau discursif plus étendu. Comme dans les textes plus anciens, le sujet zéro coïncide avec une progression à thème constant, mais les énoncés successifs sont ouverts par la mention de référents «nouveaux », non accessibles, mentionnés pour la première fois, ou absents du contexte immédiat. Une double structuration est ainsi mise en œuvre : continuité thématique sur un référent saillant, succession de prédicats relevant d'une progression «éclatée ». Ce n'est pas ici le syntagme nominal initial qu'il faut prendre en considération, mais l'ensemble du prédicat, qui renvoie à une sous- 
partie, présentée comme telle par ce type de linéarisation, d'une séquence plus large. Examinons par exemple le passage suivant, qui décrit les diverses décisions que prend le duc Loÿs de Gavre avant son départ :

23- Apres le duc appela Gerard et Gadiffer auxquels il commanda (...) D'autre part le jeune duc Louis manda et fit venir son seneschal (...) L'enfant Ypolitus fit bailler en garde à la duchesse, sa terre et son pays et tout le gouvernement bailla à Eminidus (...)

(Histoire des Seigneurs de Gavre, 1456)

[Après, le duc fit appeler (...) D'autre part, il fit appeler le jeune duc Lonis (...) Il fit donner en garde l'enfant Ypolitus (...) il donna sa terre et son pays (...) ]

Le thème constant (le duc), sujet des verbes de chaque proposition, n'est plus exprimé après la mention initiale dans la première phrase, les autres propositions du passage devant être interprétées comme des schémas : objet + verbe ; il est intéressant de noter que ce maintien de l'organisation ancienne à verbe second s'effectue alors que le marquage du sujet par le procédé de la déclinaison n'intervient pas, une forme comme le duc correspondant au sujet syntaxique dans la première phrase et à l'objet dans la deuxième.

Une construction textuelle identique peut être observée dans l'extrait suivant, emprunté au même texte :

24- La bonne dame nourrit et esleva son fils (...) Moult grand estoit et bien formé de tous ses membres; de plus bel escuyer pour lors on n'eust su trouver. Moult souefment le nourrit et esleva; maistre lui bailla pour le apprendre et endoctriner. De tables d'echecs savoit autant que nul; le cheval savoit poindre et galoper, entre dames et damoiselles se savoit deduire et esbattre (...) (id.) [La bonne dame éleva son fils (...) Il était très grand (...) on n'aurait pas pu trouver de plus bel écuyer (...) Elle l'éleva avec beaucoup de douceur (...) elle lui donna un maître pour (...) Il connaissait le jeu d'échecs (...) il savait monter à cheval (...) il savait se divertir parmi les dames (...)]

Après un enchaînement linéaire (moult grant estoit ...), deux prédicats, construits sur le schéma XV, développent le rhème initial (nourrit et esleva ...), mais on remarquera que si cette disposition syntaxique se prolonge dans les trois dernières propositions (de tables d'échecs savoit ..., le cheval savoit poindre ..., entre dames et damoiselles se savoit deduire ...), c'est avec un changement de thème, le sujet zéro renvoyant alors au fils et non plus à la dame. Tout se passe comme si la progression à thèmes dérivés - ces thèmes étant constitués, en l'occurrence, par les syntagmes verbaux - l'emportait sur la progression fondée sur le maintien d'un référent sujet, actant principal. Une étude chronologique précise permettrait de valider l'hypothèse, qu'il est naturel de formuler, de l'antériorité de cette structuration mixte sur les progressions à hyperthème qui mettent en jeu des enchaînements sur l'ensemble de la séquence.

Une étape de plus dans la structuration textuelle se trouve franchie avec l'apparition de ce que l'on pourrait appeler une « rupture thématique », une absence de 
continuité référentielle, qu'il s'agisse de relations anaphoriques interphrastiques ou de liens plus larges, au niveau de la séquence discursive. On comprend que le nouveau statut informatif du schéma SVX, qui n'est plus obligatoirement soumis au marquage de la dichotomie thème / rhème, conduise à ce type d'emploi. L'énoncé, qui doit être considéré globalement comme rhématique, comme «all focus », ne se rattache plus, même par inférence, au contexte antérieur. C'est vers la fin du XVe siècle que se développe, dans certains types de textes, cette conception d'une unité textuelle dans laquelle les marques habituelles de cohésion n'apparaissent plus comme indispensables, l'énonciation de la structure déclarative de base suffisant à établir les enchaînements attendus. On remarquera par exemple, dans les passages suivants, l'absence de mots de liaison ainsi que l'emploi de syntagmes indéfinis en fonction sujet, syntagmes qui, dans le système à verbe second, auraient été placés dans la zone postverbale, dans des séquences : circonstanciel $+\mathrm{V}+$ Sujet :

25- [Le duc, par l'espace de III heures, parla au roy d'Engleterre qui se tenoit aux champz]. Ung coulon blanc se tint sur la tente du roy l'espace de jour et demy ... (Jean Molinet, 1490)

[le duc, pendant trois heures, parla au roi d'Angleterre qui était en campagne. Un pigeon blanc se tint sur la tente du roi pendant un jour et demi ...]

26- $\quad$ Le seigneur du Fay se joindi aveuc le comte de Campebasse (...) Et porta la resolution que ilz parvenroyent legièrement à leur desir]. Beaucop de tempz se expira pour obtenir lissense pour avoir passage ...

[le seigneur du Fay s'unit au comte de Campobasso (...) Et la résolution mentionna qu'ils parviendraient sans peine ce qu'ils désiraient. Beaucoup de temps s'écoula pour que l'on puisse obtenir la permission d'avoir le passage ...]

Tout se passe comme si la simple succession des phrases, leur insertion dans le «flux discursif », suffisait pour assurer la cohérence et pour garantir la continuité du texte : le nouvel état de choses auquel renvoie l'énoncé SVX est ainsi présenté comme une sous-partie d'un tout dans lequel c'est l'ensemble de la proposition, et non plus tel ou tel référent particulier, qui joue un rôle dans la cohérence discursive. On notera toutefois que ce type d'énoncé peut également survenir en tout début de chapitre ou de nouvelle unité textuelle, pour annoncer, en quelque sorte, un changement de thème de discours, un développement sur un nouveau sujet, comme dans :

27- Ung grant abus fut semé au peuple, car une commune voix fu, qui s'esleva ... (id.)

[une grande illusion fut semée parmi le peuple, car ily eut une voix unanime qui s'éleva...]

L'enchaînement ne s'opère plus à l'intérieur d'une séquence, avec les phrases du contexte antérieur, mais avec toute l'unité discursive qui précède.

Par l'analyse de ces quelques exemples, nous avons essayé d'illustrer les spécificités du codage du dynamisme communicatif et du système des progressions thématiques dans des états de langue différents du français moderne. Dans cette description, il 
nous a semblé important d'insister sur le fait que, lorsqu'il y avait évolution, tant du côté syntaxique que du côté discursif, ce n'était pas une simple modification de surface, un simple réarrangement des constituants, une délimitation différente des zones thématiques ou rhématiques, mais une transformation plus profonde, qui affecte la conception même du niveau informatif et des règles qui en régissent l'interface avec les marques syntaxiques. Cette constatation implique quelques précautions au niveau méthodologique, afin d'éviter ce qui pourrait apparaittre comme un certain «anachronisme» des concepts utilisés. La question des unités de traitement dans lesquelles s'effectuent les opérations de présentation des référents est ainsi particulièrement cruciale, le développement des prédications secondes conduisant à discuter la pertinence d'une unité "phrase" qui aurait un statut intermédiaire entre la proposition et la période.

D'une façon plus large, c'est la conception même du texte écrit, ainsi que celle de la cohérence discursive, qui devraient être prises en considération, ce qui amènerait à s'interroger sur l'importance des conditions de production et de réception des textes - situation de dictée, lecture proche de l'oralisation - points sur lesquels la documentation est malheureusement quasiment absente. Une étude du dynamisme communicatif, fonctionnelle par nature, ne peut ignorer ces réalités. 
Bernard Combettes

\section{BIBLIOGRAPHIE}

Bauer, B., 1995, The Emergence and Development of SVO Patterning in Latin and French, Oxford University Press.

Buridant, C., 1987, «Les résidus de l'ordre OV en ancien français et leur effacement en moyen français », Romania, 108, pp. 20-65.

CHAFE, W., 1994, Discourse, consciousness, and time : the flow and displacement of conscious experience in speaking and writing, Chicago, University of Chicago Press.

COMBetTes, B.,1999, «Thématisation et topicalisation : leur rôle respectif dans l'évolution du français », in : C. Guimier (éd.), La thématisation dans les langues, Peter Lang, pp. 231-245.

COMbeTtes, B., 2000, «L'apposition comme unité textuelle et constituant phrastique: approche diachronique », Langue française 125, pp. 90-105.

Culicover, P., \& MCNally, L (ed.), 1998, The Limits of Syntax, New York, Academic Press.

ERTESCHIK-SHIR, N., 1998, "The Syntax-Focus Structure Interface», in Culicover, P. \& McNally, L. (eds), The Limits of Syntax, New York, Academic Press, pp. 211-240.

LAMBRECHT, K., 1984, Information structure and sentence form: topic, focus and the mental representations of discourse referents, Cambridge, Royaume-Uni, Cambridge University Press.

LAMBRECHT, K., 1987, « On the status of SVO sentences of spoken French ». in R. Tomlin (ed) Coherence and grounding in discourse, Amsterdam, J. Benjamins, pp. 217-262.

SKÅRUP, P., 1975, «Les premières zones de la proposition en ancien français », Revue romane, $\mathrm{n}^{\circ}$ spécial 6, Etudes romanes de l'Université de Copenhague, Akademisk Forlag. 